Mots. Les langages du politique

\title{
L'appel aux émotions dans les campagnes présidentielles. État de la recherche et approche interdisciplinaire
}

Emotional appeals in presidential campaigns. Scientific inventory and interdisciplinary approaches

El recurso a las emociones en las campañas presidenciales. Estado de la investigación y aproximación interdisciplinaria

Marion Ballet

(2) OpenEdition

Journals

Édition électronique

URL : https://journals.openedition.org/mots/22434

DOI : $10.4000 /$ mots. 22434

ISSN : 1960-600

Éditeur

ENS Éditions

Édition imprimée

Date de publication : 15 octobre 2016

Pagination : $37-44$

ISBN : 978-2-84788-850-8

ISSN : 0243-6450

\section{Référence électronique}

Marion Ballet, "L'appel aux émotions dans les campagnes présidentielles. État de la recherche et approche interdisciplinaire », Mots. Les langages du politique [En ligne], 112 | 2016, mis en ligne le 15 octobre 2018, consulté le 22 avril 2022. URL : http://journals.openedition.org/mots/22434 ; DOI : https://doi.org/10.4000/mots.22434 


\section{L'appel aux émotions dans les campagnes présidentielles. État de la recherche et approche interdisciplinaire}

Parce que la politique est fondamentalement un «partage du sensible » (Rancière, 2000), elle véhicule de très nombreux messages visant à influencer les attachements et les répulsions, les espoirs et les peurs à l'égard des objectifs, des institutions ou des acteurs de la scène publique. Les campagnes présidentielles sont des périodes particulièrement propices à l'activation d'émotions. L'enjeu primordial qui s'y joue - l'accession ou le maintien à la plus haute fonction de l'État, l'entretien des rapports de domination entre citoyens profanes et professionnels de la politique - favorise et justifie l'emploi des appels affectifs. Les émotions sont des leviers d'autant plus efficaces qu'elles sont de puissantes motivations à l'action. L'amour de la Cité, l'attachement aux institutions mais aussi la peur du désordre confortent l'allégeance des citoyens et le respect de l'ordre social.

Bien qu'au cœur des processus politiques, les émotions ont pourtant très longtemps souffert de réticences de la part des sciences sociales contemporaines ${ }^{1}$. Réputées irrationnelles, singulières et incertaines, elles étaient jugées insaisissables pour les chercheurs. S'y est ajoutée la crainte d'ébranler des méthodes et techniques d'investigation éprouvées : la volonté, à la suite de Durkheim, d'expliquer les faits sociaux comme des «choses »; la nécessité de formuler des lois tendancielles et prédictives; l'indispensable neutralité du chercheur. Très peu analysées jusqu'au début des années quatre-vingt² ${ }^{2}$, les émotions réapparaissent comme objet d'étude légitime aux États-Unis, dans un contexte de remise en

1. Avant le $20^{\mathrm{e}}$ siècle, l'étude des passions a en revanche toute sa place dans la pensée politique: Aristote, Platon, Machiavel, Descartes, Spinoza, Hume, Adam Smith, Tocqueville, etc. Voir à ce propos Ansart (1997).

2. Font exception de rares études consacrées au rôle social de certaines émotions, telles que celles menées par Erving Goffman sur les rites d'interaction (1967), de Norbert Élias sur la transformation de l'habitus affectif au cours des siècles (1969) ou de Marcel Mauss sur la place des sentiments dans les rituels funéraires (1921).

Université de Versailles Saint-Quentin-en-Yvelines, Centre d’histoire culturelle des sociétés contemporaines

marion.ballet@gmail.com 
cause du paradigme du choix rationnel3. Des controverses voient alors le jour sur la question de l'origine (biologique ou sociale) des émotions, le lien entre affect et cognition, les conventions sociales qui entourent la dénomination et l'expression des sentiments, le lien entre identité et règles émotionnelles - feeling rules (Hochschild, 1979). En France, il faut attendre le milieu des années quatrevingt-dix pour voir se développer des travaux réhabilitant la place de l'émotionnel dans la vie politique et sociale, ainsi que des thèmes de recherche renvoyant au registre affectif (Grawitz, 1985; Braud, 1991; Braud, 1996).

En ce qui concerne les campagnes électorales, deux grands types d'approches sont développées par les chercheurs. Les premières s'attachent à analyser les effets des messages émotionnels sur les électeurs, vus comme des "citoyens sentimentaux» (Marcus, 2008). Les secondes analysent la production des messages afin de comprendre les usages, par les candidats, de la «persuasion émotionnelle» (Ansart, 1983, p. 197).

\section{Le « citoyen sentimental » : approches sur les effets des messages affectifs}

Ces premières approches s’appuient sur le postulat que les émotions diffusées façonnent et alimentent les opinions politiques et les comportements électoraux. Dans la mesure où les affects ont une base cognitive, ils donnent du sens à nos actes et participent pleinement à la recherche d'information, à la constitution de jugements sociaux ou politiques et à la prise de décision (Barbalet, 2002).

La psychologie politique américaine a produit de nombreuses recherches en la matière. Certaines se sont attachées à montrer l'influence de l'image du candidat à la présidentielle sur les choix électoraux. L'ethos d'un responsable politique étant chargé émotionnellement, il influence diversement l'électorat. Certains chercheurs ont ainsi établi que l'aspect affectif joue d'autant plus que l'électeur est peu politisé et intéressé (Redlawsk, 2006). Une émotion positive, ressentie à la vue ou à l'écoute d'un candidat, suppléerait le défaut d'information et le manque de compétence politique. Elle deviendrait un motif de vote comme un autre, indiquant à l'électeur si son comportement électoral futur est susceptible de lui être favorable ou non. L'évaluation des candidats se fait alors selon une logique affective basique ("au feeling», dirait le langage commun) : Je vote pour lui ou elle car il ou elle me paraît sympathique, me rassure, etc. À

3. Connaissant un rapide essor dans les États-Unis des années soixante-dix, ce modèle influencé par les travaux d'Anthony Downs repose sur des postulats incompatibles avec la prise en compte de la dimension affective des comportements électoraux. Jugés pleinement informés et dotés de préférences, les électeurs seraient capables de hiérarchiser ces dernières et de faire le choix qui maximise le bénéfice attendu. 
l'inverse, un électeur sûr de ses orientations politiques serait moins influencé par l'image affective que produit le candidat (Ibid.).

Certaines études se sont concentrées sur la notion de charisme, la liant moins à une personnalité hors-norme qu'à une condensation, autour d'un personnage particulier, d'émotions produites par des circonstances historiques et culturelles exceptionnelles (House, Spangler, Woycke, 1991; Moscovici, 1988; Ansart, Haroche, 2007). En période de crise notamment, le leader charismatique devient le centre d'une communion affective et une identité de substitution pour des individus en perte de repère. C'est ce qui se joue en particulier dans le mythe de l'«homme providentiel» (Girardet, 1986, p. 86-95), particulièrement efficace dans les régimes présidentiels ou, comme la France, structuré autour de cette élection reine qu'est la présidentielle.

D'autres travaux ont analysé les effets particuliers de certains messages émotionnels sur les électeurs. Pour les tenants de la théorie de l'intelligence affective, inspirée des neurosciences ${ }^{4}$, un affect positif, tel que l'enthousiasme ou l'espoir, inciterait l'électeur à se fonder sur ses habitudes pour prendre sa décision politique : c'est pourquoi un parti ou un président au pouvoir aura tout intérêt à la solliciter s'il souhaite renforcer la loyauté de son électorat (Brader, 2006; Just, Crigler, Belt, 2007, p. 234-236). A contrario, la peur ou l'anxiété ébranleraient les convictions premières et inciteraient le citoyen à s'informer davantage sur le scrutin et à explorer de nouveaux points de vue. Selon cette théorie, l'électeur est d'autant plus rationnel qu'il est inquiet, car il ne peut plus s'appuyer sur ses croyances habituelles (Marcus, 2008, p. 127). La peur ayant pour effet de faire douter, elle serait ainsi plus profitable aux opposants, ou aux outsiders, qui souhaitent empêcher les citoyens de renouveler leur soutien au président en place (Schnur, 2007; Capelos, 2013). Elle serait aussi un outil pour dissuader les opposants les plus virulents de se rendre aux urnes, car si la peur n'a pas pour effet de faire changer de camp, elle pourrait en revanche favoriser l'abstention (Neuman, Marcus, Crigler, 2007, p. 368). Contrairement à la peur, la colère tendrait pour sa part à limiter la perception du risque (Lerner, Keltner, 2001) et favoriserait une prise de décision rapide et peu documentée. Elle renforcerait ainsi les positions intransigeantes, le refus de la coopération et la défense des loyautés partisanes (Huddy, Feldman, Cassese, 2007).

4. La grande majorité de ces enquêtes se fait sous forme de focus-groupes, à partir d'un corpus composé de messages issus des campagnes présidentielles américaines. 


\section{La persuasion émotionnelle : approches sur la production des messages affectifs}

Le discours politique, et a fortiori électoral, a pourvocation d'influencer l'électeur : le faire voter, mais aussi le faire croire, penser et ressentir. Cette persuasion émotionnelle prend corps dans des mots, des symboles, des paroles, des gestes, des images et, plus généralement, dans tous les procédés discursifs verbaux ou non verbaux visant à exprimer une émotion ou à solliciter chez les électeurs une réponse affective afin d'obtenir leur soutien. Les recherches présentées ci-après s'attachent moins à l'efficacité du message émotionnel qu'aux modalités de sa production : leur incarnation dans le langage, les logiques culturelles, sociales ou politiques qui les sous-tendent, les effets recherchés par le candidat (qui peuvent être différents, voire contraires aux effets émotionnels vécus par les destinataires du message).

Comme d'autres disciplines, les sciences du langage ont porté une attention de plus en plus grande aux phénomènes affectifs. À la suite des premières études rhétoriques sur le pathos, des recherches récentes se sont attachées à décrire la manière dont les émotions sont construites dans les discours et contribuent pleinement au processus argumentatif (Plantin, 2011). En d'autres termes, le discours ne sert pas uniquement à démontrer la pertinence d'une action (ce qu'il faut faire) mais aussi à légitimer un état affectif (ce qu'il faut ressentir). Certains linguistes se sont ainsi attachés, d'un point de vue éthique, à distinguer les appels affectifs « rationnels», donc acceptables, de ceux qui seraient «fallacieux», car manipulateurs (Walton, 1992).

D'autres chercheurs ont privilégié l'étude des différents procédés linguistiques à disposition des locuteurs pour exprimer, solliciter, négocier ou gérer une émotion particulière dans une interaction (Plantin, Doury, Traverso, 2000; Bazzanella, 2004; Micheli, 2010). Des catégories d'analyse ont ainsi pu être définies afin de rendre les affects à la fois intelligibles et observables dans le matériau discursif. Rapportées au discours électoral, ces études permettent de repérer les émotions véhiculées en campagne présidentielle :

- celles qui sont verbalisées par le candidat, grâce à des termes de sentiment:

Lorsqu'en qualité de maire de Paris, je me promène dans Paris, je vois les personnes âgées, j'ai toujours peur qu'elles soient agressées par un voyou. (Jacques Chirac, 12 avril 1988)

Sarkozy concentre sur sa personne l'écœurement ou la haine des victimes de la politique menée par la droite depuis cinq ans. (Nathalie Arthaud, 10 janvier 2012)

Je vous ai écoutés pour agir juste et pour tenir parole. J'ai entendu vos inquiétudes, vos colères mais aussi vos espoirs. (Ségolène Royal, 2007, profession de foi du premier tour). 
- celles qui sont représentées par des gestes, des expressions faciales (un sourire, une larme, etc.)

- celles qui sont décrites à travers les manifestations somatiques, physiologiques ou comportementales qu'elles sont supposées produire : "Le nucléaire, ça me fait terriblement frissonner» (Antoine Waechter, 12 avril 1988); «Je ne suis pas venu pleurer avec vous» (Nicolas Sarkozy, 18 décembre 2006).

- celles, enfin, qui sont montrées par un autre procédé linguistique :

J'ai été récemment visiter un hôpital à Houston, consacré aux enfants qui sont inguérissables du cancer. On m'avait demandé d'aller les distraire parce que ces pauvres gosses étaient isolés et souffraient énormément. Je suis resté pendant un moment à voir ces gosses de 5 ans, 6 ans, hurlant de douleur, avec leurs mères pleurant à leurs chevets. (Brice Lalonde, 14 avril 1981)

Je trouve honteux le mensonge des politiques qui prétendent avoir le pouvoir de changer le sort des Français alors qu'ils sont impuissants. (Marine Le Pen, 13 mars 2012)

Il convient de noter que ce type d'analyse ne permet pas de déterminer ce que ressent réellement l'émetteur du message : l'indignation exprimée peut être feinte5, la crainte simulée, la compassion non sincère.

Ces recherches linguistiques ont fourni de précieux outils méthodologiques pour des études empiriques visant le discours électoral. L'auteure du présent article a ainsi analysé les usages des appels émotionnels dans les campagnes présidentielles françaises de 1981 à 2012 (Ballet, 2012, 2014). L'objectif était de déterminer les logiques sociales et politiques qui incitent les candidats à mobiliser la peur, l'espoir, l'indignation et/ou la compassion pour influencer le vote. Le développement des techniques médiatiques, la fonction du candidat, son idéologie, sa position de sortant ou d'opposant sont autant d'éléments qui influent sur la quantité et le type d'affects sollicités.

D'un point de vue diachronique, les candidats sont de plus en plus enclins à susciter l'émotion de leurs électeurs. La hausse du nombre d'appels affectifs recensés dans les discours de 1981 à 2012 en témoigne : de plus en plus contraints de "faire court» pour être efficaces, soucieux d'apparaître au plus proche de leurs électeurs (Le Bart, Lefebvre, 2005), les candidats ont intériorisé la nécessité de « faire ressentir l'émotion, [d']aller au nerf» (Saussez, 2004, p. 233). S'impose ainsi en quelques années l'idée que la parole politique n'est plus à même, seule, d'emporter l'adhésion.

Une autre évolution à noter concerne le type de registres utilisés par les candidats (Ballet, 2012, p. 41-64). Fortement imprégnés de peur en période de guerre froide (1981-1988), les discours électoraux se teintent par la suite de

5. Que l'on songe à la controverse qu'a provoquée la «juste colère » (feinte ou sincère ?) exprimée par Ségolène Royal lors du débat de l'entre-deux tours de l'élection présidentielle de 2007. Pour une analyse « genrée » des usages de la colère lors de ce débat, voir le passionnant article d'Achin et Dorlin (2008). 
compassion (1995-2012). À l'heure où la figure de la «victime» s'impose dans les journaux télévisés, les documentaires et même les émissions dites de divertissement (Erner, 2006), les candidats à la présidentielle font de plus en plus état de leur inclination à partager les maux et souffrances de leurs électeurs : ils s'adressent «à ceux qui se sentent tirés vers le bas» (Ségolène Royal, 30 avril 2007) et « qui ne croient plus en rien» (Nicolas Sarkozy, 15 février 2012). Le contexte sécuritaire anxiogène de plus en plus prégnant dans notre pays, confronté aux attentats de janvier et novembre 2015 (Boucheron, Robin, Payre, 2015), laisse présager un retour de la peur dans les discours électoraux, hypothèse qu'il conviendra de mettre à l'épreuve de la prochaine présidentielle.

Cette analyse s'est aussi nourrie de la sociologie des mouvements sociaux pour appréhender le rôle des affects dans les cultures politiques et les engagements partisans. L'un de ses courants, emmené par Jeff Goodwin et James M. Jasper, offre en effet des éclairages précieux sur le travail de légitimation des affects, opéré pour mobiliser, former, fidéliser les individus et donner une identité collective au groupe militant (Goodwin, Jasper, 2004; Traïni, 2009, 2015). Les appels à la colère dans les discours d'extrême gauche, communistes et trotskistes, visent par exemple cet objectif (Ballet, 2012, p. 91-104). Il s'agit, d'une part, de rappeler au groupe ce qui fonde sa culture politique, ses valeurs, sa force, ses desseins et ainsi d'unifier, dans un même élan émotionnel, un électorat parfois très divers : «les gens qui ont raison de protester, d'exprimer leur révolte» (Robert Hue, 11 avril 1995) ou «ceux qui sont mécontents [et] ceux qui luttent » (André Lajoinie, 11 avril 1988). Il s'agit, d'autre part, de tourner les électeurs/militants vers l'action. Minimisant la perception du risque, la colère favorise le passage à l'acte (Huddy, Feldman, Cassese, 2007, p. 208) : elle nourrit donc la passion révolutionnaire et peut être très efficace, en campagne électorale, si les discours parviennent à la canaliser dans le vote. L'électeur est ainsi invité à «faire passer sur ces élections le souffle de leur colère et de leur amertume» (Arlette Laguiller, 12 avril 1988). Au-delà de cet exemple particulier, il a été possible d'identifier les marqueurs émotionnels propres à chaque culture politique et véhiculés dans les discours de leurs candidats : la peur pour les écologistes, un mélange de peur et d'indignation pour les frontistes, l'espoir pour les cultures de gouvernement - gauche et droite modérées (Ballet, 2012, p. 91-173).

En quelques décennies, les émotions sont redevenues des objets de recherche légitimes. Cette évolution a fait naitre des perspectives particulièrement fécondes dans l'analyse des discours politiques et a fortiori des discours de campagne présidentielle. Qu'elles s'attachent à déterminer les effets des appels émotionnels sur les électeurs ou les logiques qui sous-tendent la production des messages affectifs par les candidats, les recherches présentées ci-dessus battent définitivement en brèche l'idée de comportements politiques purement rationnels : "La démocratie, c'est bien sûr donner sa voix, mais on ne la donne jamais "dans le silence des passions" ( (Roy, 1999, p. 585). 


\section{Références}

ACHIN Catherine, Dorlin Elsa, 2008, « Nicolas Sarkozy ou la masculinité mascarade du Président», Raisons politiques, $\mathrm{n}^{0} 31$, p. 19-45.

ANSART Pierre, 1983, La gestion des passions politiques, Lausanne, L'Âge d'Homme, p. 197.

- 1997, Les cliniciens des passions politiques, Paris, Le Seuil.

ANSART Pierre, HAROCHE Claudine, 2007, Les sentiments et le politique, Paris, L'Harmattan.

BALlet Marion, 2012, Peur, espoir, indignation, compassion. L'appel aux émotions dans les campagnes présidentielles (1981-2007), Paris, Dalloz.

- 2014, Émotions et élections. Les campagnes présidentielles françaises (1981-2012), Paris, INA.

BARBALET Jack, 2002, Emotions and Sociology, Oxford, Blackwell.

Bazzanella Carla, 2004, "Emotions, language, context», Emotion in Dialogic Interaction. Advances in the Complex, E. Weigand éd., Londres, Benjamins, p. 59-76.

Boucheron Patrick, Robin Corey, PAYre Renaud, 2015, L'exercice de la peur. Usages politiques d'une émotion, Lyon, Presses universitaires de Lyon.

Brader Ted, 2006, Campaigning for Hearts and Minds. How Emotional Appeals in Political Ads Work, Chicago, The University of Chicago Press.

Braud Philippe, 1991, Le jardin des délices démocratique. Pour une lecture psychoaffective des régimes pluralistes, Paris, Presses de la FNSP.

- 1996, L'émotion en politique. Problèmes d'analyse, Paris, Presses de Sciences Po.

CAPElos Tereza, 2013, "Understanding anxiety and aversion. The Origins and consequences of affectivity in polical campaigns », Emotions in Politics. The Affect Dimension in Political Tension, N. Demertzis éd., Palgrave Macmillan, p. 39-58.

ElıAs Norbert, 1969, Die höfische Gesellschaft, Neuwied, Luchterhand.

ERnER Guillaume, 2006, La société des victimes, Paris, La Découverte.

GIRARdet Raoul, 1986, Mythes et mythologies politiques, Paris, Le Seuil.

GoffMAn Erving, 1967, Interaction Ritual. Essays on Face-To-Face Behavior, New York, Doubleday.

GoodWIN Jeff, JASPER James M., 2004, Rethinking Social Movements. Structure, Meaning, and Emotion, Lanham, Rowman and Littlefield.

Grawitz Madeleine, 1985, "Psychologie et politique», Traité de science politique, M. Grawitz, J. Leca éd., Paris, PUF, t. 3, p. 1-139.

HochSCHILD Arlie R., 1979, «Emotion work, feeling rules, and social structure », American Journal of Sociology, vol. 85, nº3, p. 551-575.

House Robert J., SPANGLER William D., WoYCKE James, 1991, «Personality and charisma in the U.S. Presidency. A psychological theory of leader effectiveness », Administrative Science Quarterly, vol.36, n³, p.364-396.

Huddy Leonie, Feldman Stanley and CASSESE Erin, 2007, "On the distinct political effects of anxiety and anger», The Affect Effect. Dynamics of Emotion in Political Thinking and Behavior, W. R. Neuman, G. E. Marcus, A. Crigler, M. MacKuen éd., Chicago, University of Chicago Press, p. 202-230. 
JUSt Marion R., CRIgLER Ann N., Belt Todd, 2007, «Don’t give up hope. Emotions, candidate appraisals and votes ", The Affect Effect. Dynamics of Emotion in Political Thinking and Behavior, W. R. Neuman, G. E. Marcus, A. Crigler, M. MacKuen éd., Chicago, University of Chicago Press, p. 231-259.

Le Bart Christian, Lefebvre Rémi éd., 2005, Mots. Les langages du politique, nº77, «Proximité».

LERNER Jennifer S., KELTNER Dacher, 2001, «Fear anger, and risks , , Journal of Personality and Social Psychology, vol. 81, $\mathrm{n}^{\circ}$ 1, p. 146-159.

MARCUS George E., 2008, Le citoyen sentimental. Émotions et politique en démocratie, Paris, Presses de Sciences Po.

MAuss Marcel, 1921, «L'expression obligatoire des sentiments (rituels oraux funéraires australiens) », Journal de psychologie normale et pathologique, vol. 18, p. 425-434.

Michel Raphael, 2010, L'émotion argumentée. L'abolition de la peine de mort dans le débat parlementaire français, Paris, Le Cerf.

Moscovıc Serge, 1988, La machine à faire des dieux. Sociologie et psychologie, Paris, Fayard.

Neuman Russell W., Marcus George E., Crigler Ann N., 2007, The Affect Effect. Dynamics of Emotion in Political Thinking and Behavior, Chicago, Londres, The University of Chicago Press.

Plantin Christian, 2011, Les Bonnes raisons des émotions. Principes et méthode pour l'analyse de la parole émotionnée, Berne, Peter Lang.

Plantin Christian, Doury Marianne, Traverso Véronique, 2000, Les émotions dans les interactions, Lyon, Presses universitaires de Lyon.

RANCIÈrE Jacques, 2000, Le partage du sensible : esthétique et politique, Paris, La Fabrique.

Red LAWsK David P., 2006, Feeling politics. Emotion in Political Information Processing, New York, Palgrave.

RoBIn Corey, 2004, Fear. The History of a Political Idea, Oxford, Oxford University Press.

Roy Olivier, 1999, "Le contrat social ne se fait pas dans le silence des passions», Critique. Revue générale des publications françaises et étrangères, vol.55, $\mathrm{n}^{\circ} 625$ 626, p. 581-586.

SAUSSEZ Thierry, 2004, Le style réinvente la politique, Paris, Presses de la Renaissance.

SCHNUR Dan, 2007, "The affect effect in the real world of political campaigns", The Affect Effect. Dynamics of Emotion in Political Thinking and Behavior, W. R. Neuman, G. E. Marcus, A. Crigler, M. MacKuen éd., Chicago, University of Chicago Press, p. 357-374.

TRAïNI Christophe, 2009, Émotions... Mobilisations!, Paris, Presses de Sciences Po.

- 2015, Émotions et expertises. Les modes de coordination de l'action collective, Rennes, Presses universitaires de Rennes.

Walton Douglas, 1992, The Place of Emotion in Argument, Pennsylvania, Penn State University Press. 